\title{
Health and health behaviours among a cohort of first year nursing students in Scotland: A self-report survey
}

Josie MM Evans MA (Oxon) MPH PhD, Associate Professor of Public Health (josie.evans@stir.ac.uk)

Claire E Eades MSc, Lecturer (c.e.eades@stir.ac.uk)

Dawn M Cameron PhD, Lecturer (dawn.cameron@stir.ac.uk)

Faculty of Health Sciences and Sport, University of Stirling, Scotland, UK, FK9 4LA

Correspondence to: Dr Josie MM Evans PhD, Faculty of Health Sciences and Sport, University of Stirling, Stirling, Scotland, UK, FK9 4LA

Tel: 01786-466352.

Fax : 01786-466333

E mail: josie.evans@stir.ac.uk

Overall word count (including Abstract and References): 4,073

Acknowledgments/Funding: This study was funded by the Faculty of Health Sciences and Sport, University of Stirling. Thank you to all the students who voluntarily participated in this survey.

Accepted refereed manuscript of:

Evans JMM, Eades CE \& Cameron DM (2019) Health and health behaviours among a cohort of first year nursing students in Scotland: A self-report survey. Nurse Education in Practice, 36, pp. 71-75 DOI: https://doi.org/10.1016/j.nepr.2019.02.019

(C) 2019, Elsevier. Licensed under the Creative Commons Attribution-NonCommercial-NoDerivatives 4.0 International http://creativecommons.org/licenses/by-nc-nd/4.0/ 


\begin{abstract}
The study aim was to collect data on the health and health-related behaviours of undergraduate nursing students at a Higher Education Institution in Scotland, to identify the need for potential health behaviour change interventions as part of their undergraduate course. An anonymous self-report questionnaire (with questions about physical activity, diet, sleep, alcohol, smoking, mental health) was administered to first year nursing students at a Scottish university. The response rate was 88\%, with 207 respondents ( 26 male, 178 female, 3 other). Age ranged from 16-45 years (mean 24.5 years). Overall, 48 (23.1\%) students rated their physical health as excellent/very good, and $100(48.3 \%)$ their mental health as such. $157(76.2 \%)$ students were achieving 150 minutes of physical activity per week. There were 48 (29\%) and 30 (18.2\%) overweight and obese students respectively. 129 (62.6\%) students viewed a mobile device for $>30$ minutes before sleep. $176(86.3 \%)$ students consumed alcohol, with 32 (15.4\%) reporting binge drinking. The prevalence of current smoking was $24.8 \%$. The students' health behaviour profile was therefore broadly similar to that of the general population in Scotland, but smoking, diet, sleep practices and binge-drinking were identified as priority areas for health education and intervention.
\end{abstract}




\section{Highlights}

- This health behaviour survey of first year undergraduate nursing students achieved $88 \%$ response rate.

- One quarter of the students smoked, and nearly half (47\%) were overweight or obese.

- Binge-drinking was reported by $15.4 \%$.

- Nearly two thirds of students looked at a mobile device for $>30$ minutes before sleep.

- Smoking, diet, sleep practices and binge-drinking were identified as priority areas.

\section{Keywords}

Nursing; education; health behaviour; behaviour change 


\section{Introduction}

As the Scottish Government has highlighted, the health-promoting behaviours, and the health and wellbeing of nurses, are coming under increasing scrutiny, with recent evidence suggesting that their lifestyle behaviours and their physical and mental health are poorer than those of other allied health professionals, and even those of the general population (Scottish Government, 2017). There are two reasons why this is a concern. First, nurses of today will have longer careers than their predecessors. It is important that they maintain the physical and psychological capacity to provide the high standards of nursing care that are required in today's complex health care systems. Second, the public health roles of nurses are expanding and developing, with more involvement in primary prevention and health promotion; and it has been shown that nurses who have healthy lifestyles themselves are more likely to be successful at promoting health among their patients (McDowell et al., 1997; Radsma and Bottorff, 2009; Hicks et al, 2008).

In Scotland and elsewhere, there is a commitment to an all-graduate nursing workforce. During their University degrees, future nurses acquire the academic knowledge to practise health promotion. It has been argued that exposure to this may have a positive effect on their health behaviours, but conversely the academic and social demands of University life may have a negative effect, with the health behaviours of some students deteriorating during this time (Bryer et al., 2013). There have been several surveys in various countries, providing a mixed picture of the lifestyles of undergraduate nursing students. For example, a study among 140 first year nursing students in Spain and Colombia showed that fewer than 1 in 10 smoked and alcohol consumption was moderate, and but that the prevalence figures for poor diet, poor sleep and insufficient physical activity were high (Rodriguez-Gasquez et al., 2016). In contrast, 20\% of 182 first and third year undergraduate students in Ireland smoked, and 19\% of the females drunk more alcohol than the recommended weekly limit, but their physical activity levels were more favourable (Burke and McCarthy, 2011). Other studies in Iran, Hong Kong and Thailand have highlighted generally poor levels of health promoting lifestyles among nursing students (Rezaei-Adaryani and Rezaei-Adaryani, 2012), and low levels of physical activity and fitness (Choi Hui, 2002; Klainin-Yobas et al., 2015)

There are now increasing numbers of non-traditional students registering on undergraduate nursing programmes. Non-traditional students do not enter into higher education immediately 
after leaving school and are therefore older, often with dependents. Evidence from the United States suggests that the health behaviours of non-traditional students may be poorer than those of traditional students, and that they may be more resistant to behaviour change, with 70 nontraditional nursing students scoring lower on a Health Promoting Lifestyle Profile than 40 traditional students, but higher on a Barriers to Health Promotion Activities instrument (Bryer et al., 2013).

It seems evident that interventions to develop and encourage healthy behaviours among the nurses of the future should be developed and implemented while they are still easily identifiable as a captive audience at an educational institution. However, international evidence indicates that priority areas for health promotion are likely to be culture and country-specific, and different interventions may be needed in different countries, tailored to the specific group in question. The SHOTS study (Student Health Over Time in Stirling) is an ongoing series of self-report questionnaires designed to collect data on the health behaviours of undergraduate nursing students at the University of Stirling, Scotland, at three points during their course (at the start of their first, second and third years). The aim of the study is to identify priority areas for health behaviour change interventions and also to document how health behaviours change among nursing students over time. In this paper, we report on the health behaviours of the 2016 cohort at the start of their course, and also compare these behaviours between traditional and non-traditional students. 


\section{Methods}

A self-report paper questionnaire was administered to first year undergraduate nursing students (mental health and adult branch) during a timetabled session in their induction week by a member of the research team (all academic staff at the institution), after they had received Participant Information Leaflets study two days previously. The questionnaire was adapted from one used among all students (at any level or subject) at a different Higher Education Institution (HEI) institution as part of a Healthy University Project, and contained seven sections relating to physical activity, eating habits, sleep, alcohol, smoking, mental health and demographics, with a combination of open and semi-structured questions. This original questionnaire was adapted from sections of questionnaires used in several other HEIs and was also informed by the Scottish Health Survey (Scottish Government, 2016). It had significant stakeholder input (Deniozou, 2015). Within the questionnaire, mental health was assessed using the Warwick-Edinburgh mental wellbeing scale (WEMWBS) (Warwick Medical School, 2018). Body mass index (BMI) was also determined by asking students to report their weight and height in any units, then converting this to $\mathrm{kg} / \mathrm{m}^{2}$.

The questionnaires were anonymous. The students were asked to provide a 12-digit alphanumeric identifier on their questionnaire but not to give any information that would make them identifiable. The identifier comprised year of intake, code for adult or mental health, month of birth, number of letters in name, number of siblings, and first letters of parents' given names. It was designed to enable questionnaires completed by the same student in subsequent years to be linked together, without rendering them identifiable. For the purposes of this study, students were classified as non-traditional students if they (or any of their siblings) were the first person to go to University in their family and they were also aged over 24 years. If any students did not wish to complete the questionnaire they were advised to return blank forms at the end of the timetabled session.

Data were analysed using the SPSS v21.0 programme (Chicago, Illinois). All variables were checked for outliers. In general, for continuous variables, mean, median, standard deviation and range were determined. Proportions were calculated for categorical variables. Statistical tests used, as appropriate, included $\mathrm{t}$ tests for comparison of means and Chi-squared tests for proportions between traditional and non-traditional students. We also carried out sub-group analyses, comparing BMI among students with different health behaviours (whether they 
carried out 150 minutes of physical activity per week, whether they had breakfast most days and whether they ate 5 portions of fruit and vegetables per day). Finally, we compared behaviours among undergraduate nursing students with those of the general population, by accessing results from the 2016 Scottish Health Survey (Scottish Government, 2016). This is an annual survey of a representative sample of the general population living in private households in Scotland, with the 2016 round producing data on 4,323 adults.

Ethical approval for the study was granted by the University of Stirling School of Health Sciences Research Ethics Committee in July 2016 (reference 15/16:67).

\section{Results}

Completed questionnaires were returned by 207 students ( 26 male, 178 female, 2 non-binary, 1 not given). This gave a response rate of $88 \%$ from a cohort of 235 students. Age of the students ranged from 16 to 45 years, with a mean age of 24.5 years (median 22 years). 126 students stated that either they (or a sibling) were the first person to go to University in their family, of whom 50 were also aged $>24$ years and classified as non-traditional students (42 female). Table 1 provides summary results for the health behaviour domains stratified by traditional or non-traditional student status. Overall, 48 (23.1\%) students rated their physical health as excellent or very good, $100(48.3 \%)$ as good, and $58(28.0 \%)$ as fair or poor. For general mental health, the equivalent figures were 100 (48.3\%), $83(40.1 \%)$ and $24(11.6 \%)$. The results of chi squared tests comparing proportions of traditional and non-traditional students achieving 150 minutes of physical activity per week, proportions overweight and obese, and proportions currently smoking are also shown in Table 1; as are the results of a ttest examining differences in mean sedentary time and mean BMI between traditional and non-traditional students.

\section{Physical Activity}

Table 1 shows that 157 (76.2\%) students were achieving 150 minutes of physical activity (PA) per week in total, 74 of whom were managing this with 30 minutes of PA five or more days of the week. Sedentary time among 190 students ranged from 30 minutes to 17.5 hours on a weekday (mean 6.0; median 6 hours), and from 1 hour to 20 hours at the weekend (mean 6.4; median 6 hours). There was no evidence that non-traditional students were less likely to be achieving 150 minutes of PA; $78 \%$ reported achieving this compared to $75.6 \%$ of traditional 
students, and they had statistically significant lower levels of sedentary time, particularly at the weekend. Table 2 shows that slightly higher proportions of students were accumulating 150 minutes of PA per week compared to SHS respondents of the same age and sex.

\section{Diet}

Table 1 shows that $50(24.2 \%)$ students rated their diet as very healthy or healthy, $121(58.5 \%)$ as average, and $36(17.4 \%)$ as unhealthy or very unhealthy. $142(68.6 \%)$ students reported having breakfast most days, $25(12.1 \%)$ as having five or more pieces of fruit and vegetables per day, and $88(42.7 \%)$ as having a takeaway meal at least weekly. The respective proportions among non-traditional students were $68 \%, 14 \%$ and $50 \%$. However, lower proportions of students were consuming five or more portions of fruit and vegetables per day compared to SHS respondents of the same age and sex (Table 2).

\section{Body Mass Index (BMI)}

BMI (in $\mathrm{kg} / \mathrm{m}^{2}$ ) was available for 165 students, among whom the mean (median) BMI was 25.2 (24.1) (Table 1). However, 48 (29\%) of these students were classified as overweight and 30 $(18.2 \%)$ as obese. The proportions within age and sex sub-groups were not very different from those of SHS respondents (Table 2). Mean BMI was 24.9 among traditional students and 26.1 among non-traditional students, but the difference was not statistically significant $(\mathrm{t}=-1.35$; $\mathrm{p}=0.181)$.

Mean BMI (in $\mathrm{kg} / \mathrm{m}^{2}$ ) was slightly lower (24.8) among students who achieved 150 minutes of PA per week, compared to those who did not meet this recommendation (26.5) but again the difference was not statistically significant $(\mathrm{t}=-1.71 ; \mathrm{p}=0.09)$. Similarly, it was slightly lower among students who reported eating breakfast most days, and those who ate more than five pieces of fruit/veg per day. However, there was a statistically significantly higher BMI among students who reported having takeaway meals at least weekly (26.9), compared to those who had less frequent takeaway meals $(24.0)(\mathrm{t}=-3.67 ; \mathrm{p}=0.01)$.

\section{Sleep}

$106(51.2 \%)$ students reported sleeping very well or well, $79(38.2 \%)$ as average, and 23 $(11.1 \%)$ as badly or very badly. $70(33.8 \%)$ had less than seven hours sleep per night. 129 (62.6\%) students reported looking at a mobile device for more than half an hour in bed before 
going to sleep. Among non-traditional students, the respective proportions were $22 \%$ and $54 \%$ (Table 1) .

\section{Alcohol}

Table 1 shows that $176(86.3 \%)$ students reported drinking alcohol, of whom four drunk every day or most days of the week. $134(65 \%)$ students stated they drunk only occasionally or never drunk. Of the $70(39.8 \%)$ students who reported drinking at least weekly, the mean number of units consumed per week was 8.99 among 59 who provided a response. However, 13 students (10\% of 126 students for whom data were available) reported drinking 14 or more units per week. This proportion was lower than among SHS respondents (Table 2). Out of all students, 32 reported binge drinking (more than 6 or 8 units in a single occasion) at least weekly. Patterns of drinking among non-traditional students were very similar to those of the traditional students. $42(84 \%)$ reported drinking alcohol, but $32(64 \%)$ drunk only occasionally or never drunk. Of the 14 (28\%) students who reported drinking at least weekly, the mean number of units consumed per week was 8.71.

\section{Smoking}

Fifty (of 202) students reported current smoking, 32 of whom smoked every day. This prevalence of $24.8 \%$ for current smoking was similar to the prevalence of $26 \%$ among nontraditional students (Table 1). The prevalence figures were also similar within age and sex subgroups compared with the SHS (Table 2).

\section{Mental Health}

Among 198 students, the WMWBS score ranged from 17.8 to 70. The mean score was 50.9 which accords with the mean score for the general population, and also those for age and sex sub-groups in the SHS (Table 2). There were 29 (14.6\%) traditional students and 9 (18.4\%) non-traditional students who had scores that were classified as below-average (more than one deviation below the mean score). 


\section{Discussion}

Despite concerns over the health and health behaviour measures among the nursing profession (Scottish Government, 2017), this study provides no evidence that these are less favourable among a cohort of first-year nursing students in Scotland, than among respondents of the same age to the Scottish Health Survey (SHS) in 2016. While the samples may not be directly comparable in that SHS respondents may come from a wider range of social backgrounds than those of the nursing students, it seems that nurses start their nursing training in Scotland with a fairly typical health behaviour profile. So if nurses do have poorer health and lifestyle than other professionals in Scotland, these must diverge over the course of their subsequent training and career.

There were two exceptions to this. The dietary measure common to both surveys was reported number of portions of fruit and vegetable consumed per week, with a lower proportion of nursing students achieving at least five portions than SHS respondents. This might be explained by many nursing students living away from home for the first time and being responsible for their own diet. The other notable difference between student nurses and SHS respondents was for self-rated health. A lower proportion of students rated their general physical health as very good or excellent than respondents to the SHS rated their general health as very good. While the scale and the wording of the questions were not directly comparable, these differences were substantial, although it is possible that they arise as nursing students become more conscious of their own health status. The mean WMWBS scores, which is an indicator of mental health, were similar among nursing students and the SHS sample (Table 2).

Health behaviour measures among non-traditional students were no worse than those of traditional students. Although other studies have used a broader range of variables to establish the status of individual students (age, sex, whether part-time or full-time, ethnic background, English as a second language and dependent children) (Bryer at al., 2013), these variables are were not alll available or relevant to this Scottish cohort. We were able to use only age, and whether the student was a first generation University attender. So although we found few statistically significant differences between traditional and non-traditional students in contrast to a study among American nursing students (Bryer et al., 2013), a larger study with a more sensitive method of differentiating by social background (and adjusting for other confounding variables) would have more power to detect differences if they existed. 
There is clearly no room for complacency. While student nurses may be typical of the Scottish general population, it is well known that Scotland has poorer health, shorter life expectancy and higher prevalence of damaging health behaviours than other industrialized countries (Gray, 2007). This means that student nurse behaviours may be worse than those of student nurses in other countries. For example, recent surveys suggest that this cohort of nursing students are more than twice as likely to smoke as those in Spain and Colombia (Rodriguez-Gasquez et al., 2016), with smoking prevalence also lower (21\%) among 381 student nurses in Australia (Walsh et al., 2012); although one study in Spain study reported similar smoking prevalence to Scotland among nursing and physiotherapy students (Pericas et al., 2009). However, some caution should be exercised when making comparison between different studies using different survey questions and methods.

Results for alcohol consumption were interesting in that around two-thirds of students reported drinking alcohol never or only occasionally. This proportion was actually higher than among Spanish nursing students (Rabanales Sotos et al., 2014), However, among those students who did consume alcohol, while the mean number of units consumed per week was lower than among respondents to the SHS survey (which was 16.9 among all respondents who had consumed any alcohol in the previous year) (Scottish Government, 2016), there was a relatively high frequency of binge drinking (more than 8 or 6 units on a single occasion for men and women). This pattern of drinking is not unusual among University students, but needs to be addressed given its risks for the student and possibly even patients. The physical activity profiles of this cohort of students were more favourable, and higher proportions met recommended levels than among 193 nursing students (from all years) at a UK University (Blake et al., 2016), as did a sample from Ireland (Burke and McCarthy, 2011).

The survey has highlighted other target areas for health behaviour change interventions. For example, as well as fruit and vegetable consumption, other indicators of diet were worrying, with a high proportion of students consuming takeaway meals frequently (especially as this was associated with higher BMI), and nearly one third skipping breakfast on some days. Nearly half of all the students for whom we were able to calculate BMI were already either overweight or obese. The prevalence of overweight / obesity is 69\% among all Scottish nurses (Kyle et al., 2016). This is a priority area for health promotion if nurses are required to be role models for addressing the current obesity epidemic. 
The study has uncovered some poor sleeping practices (this was not measured in the SHS), with around one-third of students having fewer than seven hours sleep a night, and nearly two thirds looking at a mobile device for more than half an hour in bed before sleep. This is a concern given the association between short sleep and higher mortality and chronic disease levels (Itani et al., 2016), with high levels of screen time before sleep associated with poor sleep quality (Christensen et al., 2016).

The sample size in this study was small. It was restricted to one University in Scotland (of four undergraduate nursing providers) and comparisons with the SHS among very small age and sex sub-groups of the population (particularly among men) must be interpreted with caution. We were also only able to compare variables which were collected in both datasets and present these descriptively to highlight possible areas of concern (given the small numbers). However, there was a very high response rate so we can argue that the results are representative of the entry cohort; we achieved $88 \%$ coverage of the entire first year. One limitation is the self-report nature of the data collected, with no objective means of verifying the results. It is possible that social desirability bias influenced students' reporting of their health behaviours, whether this arises through self-deception or as a conscious wish for them to present a favourable image of themselves. While this would be the case for any self-report survey, we aimed to minimise the bias by stressing to the students that all data were anonymous and no attempt would be made to identify them as individual. It is also important to recognise that the study was crosssectional and that any identified associations cannot be interpreted as causal. A final limitation was the relatively blunt method of categorising students as non-traditional or traditional.

In conclusion, while this study indicates that a cohort of first year nursing students in Scotland has a health behaviour profile broadly similar to that of the general population in Scotland, we would argue that smoking, diet and sleep practices may be priority areas for health education and intervention in this particular cohort. There may also be subgroups in the cohort who are at risk of harmful drinking (specifically binge drinking) who would benefit from targeted interventions. Further work is ongoing to identify whether health behaviour change interventions relating to these priority areas can be delivered as part of the undergraduate curriculum. 


\section{References}

Blake H, Stanulewicz N, McGill F. Predictors of physical activity and barriers to exercise in nursing and medical students (2016). JAN; 73:917-929.

Bryer J, Cherkis F, Raman (2013). Health-promotion behaviours of undergraduate nursing students: A survey analysis. Nursing Education Perspectives; 410-415.

Burke E, McCarthy B (2011). The lifestyle behaviours and exercise beliefs of undergraduate student nurses: A descriptive study. Health Education; 111:230-246.

Choi-Hui WH (2102). The health-promoting lifestyles of undergraduate nurses in Hong Kong. J Prof Nursing; 18:101-111.

Christensen MA, Bettencourt L, Kaye L, Moturo ST, Nguyen KT, Olgin JE, Pletcher MJ, Marcus GM (2016). Direct measurements of smartphone screen-time: Relationships with demographics and sleep. PLOS One 11: e0165331

Deniozou T (2015). Student Health and Lifestyle Survey. Healthy University Project. University of Edinburgh

https://www.ed.ac.uk/files/atoms/files//healthy_university_research_report_final_july_15.pdf .Accessed 11/1/2018).

Gray L (2007). Comparisons of health-related behaviours and health measures between Glasgow and the rest of Scotland. Glasgow Centre for Population Health, Glasgow. http://eprints.gla.ac.uk/132261/ Accessed 16/1/2018

Itani O, Jike M, Watanabe N, Kaneita Y (2016). Short sleep duration and health outcomes: a systematic review, meta-analysis, and meta-regression. Sleep Med 32:246-256.

Klainin-Yobas P, He H-G, Lau Y. Physical fitness, health behaviour and health among nursing students: A descriptive correlational study. Nurse Education Today; 35:1199-1205.

Kyle RG, Neall RA, Atherton IM (2016). Prevalence of overweight and obesity among nurses in Scotland: A cross-sectional study using the Scottish Health Survey. Int J Nursing Studies; $53: 126-133$

McDowell N, McKenna J, Naylor PJ (1997). Factors that influence practice nurses to promote physical activity. Br J Sports Med; 31:308-313.

Pericas J, Gonzalez S, Bennasar M, De Pedro J, Aguilo A, Bauza L (2009). Cognitive dissonance towards the smoking habit among nursing and physiotherapy students at The University of Balearic Islands in Spain. International Nursing Review 56; 95-101. 
Rabanales Sotos J, Lopez Gonzalez A, Parraga Martinez I, Campos Rosa M, Simarro Herraez MJ, Lopez-Torres H (2015). Prevalence of hazardous drinking among nursing students. JAN; 71:581-590.

Radsma J, Bottorff JL (2009). Counteracting ambivalence: nurses who smoke and their health promotion role with patients who smoke. Res Nurse Health 32: 443-452.

Rezaei-Adaryani M, Rezaei-Adaryani M (2012). Health-promoting lifestyle of a group of Iranian medical, nursing and allied health students. J Clin Nurs ; 21:3587-3589.

Rodriguez-Gasquez M, Chaparro-Hernandez S, Gonzalez-Lopez JR (2017). Are first year students' lifestyles coherent with their future career? Int J Nurs Pract; 23:e12511.

Scottish Government (2016). Scottish Health Survey 2016. Volume 1: Main Report. http://www.gov.scot/Publications/2017/10/2970/downloads\#res-1 Accessed 16/1/2018.

Scottish Government (2017).Nursing 2030 Vision: Promoting confident, competent and collaborative nursing for Scotland's future

Walsh RA, Cholowski K, Tzelepis F, Stojanovski OE (2012). Smoking prevalence, attitudes and confidence about tobacco roles among Australian nursing students. JAN; 23:181-190.

Warwick Medical School (2018). Uses of WEMWBS. https://warwick.ac.uk/fac/med/research/platform/wemwbs/researchers/uses/Accessed $\underline{17 / 1 / 2018}$. 